\title{
Characterization of unsaturated response of shale-like materials
}

\author{
Hyunbin Kim $^{1}$ and Roman Y. Makhnenko ${ }^{1}$ \\ ${ }^{1}$ Department of Civil and Environmental Engineering, University of Illinois at Urbana-Champaign, Urbana, IL, 61801, USA
}

\section{Introduction}

Tight shale-like formations are often considered as barriers for fluid flow in geo-energy applications. The water retention-related mechanisms of the material, which are directly related to the sealing capacity of the tight formations, vary with physical, thermal, and chemical disturbances over time. The behavior of fully saturated shales can be described by the poroelastic framework, but the unsaturated poroelastic properties are generally difficult to be measured in the laboratory since the solid, pore, and fluid compressibility cannot be neglected. Recently, the poroelasticity theory has been extended from saturated to unsaturated materials using the micromechanical analysis in which macroscopic constants are calculated based on microscale measurable properties under the fully saturated condition and the water retention curve (Cheng 2019). This extended model intends to estimate the bulk material constants at a given degree of saturation without the need for time-consuming experiments on low permeable shales. In this study, intact and remolded shaly facies of Opalinus Clay from Mont Terri underground rock lab in Switzerland are characterized, and measurable macroscopic properties are compared with the model prediction.

\section{Constitutive Model for Unsaturated Rock}

The contributions of wetting and nonwetting fluids to the linear stress-strain relations in Biot poroelastic theory have been recently considered by Cheng (2019). Unsaturated porous media where wetting and nonwetting fluid exist simultaneously in voids do not adhere to the behavior of linear poroelastic materials since the interaction between the fluids and solid is highly related to the diffusion process of pore fluids. In the macroscopic constitutive model for unsaturated poroelastic materials, total pore fluid pressure is assumed as the sum of the products of the degree of saturation of each fluid and its pressure. Biot effective stress is thus affected by both fluids' pressures. Another difference between the models for saturated and unsaturated materials is that a variation in the volumetric content of one pore fluid is proportional to its pressure, as well as the pressure of the other pore fluid since the injection will cause pressure changes in both fluids. The microscopic analysis adopts the one pore channel assumption, meaning that pore fluids compete for the space in a channel passing through the representative element volume in compliance with the surface tension between the fluids (Figure 1). This assumption gives the direct correlation between the microscale and macroscale variations in pore fluids, such that it is possible to calculate the macroscale material properties based only on the microscale constants obtained for the fully saturated condition, porosity, and the retention curve.

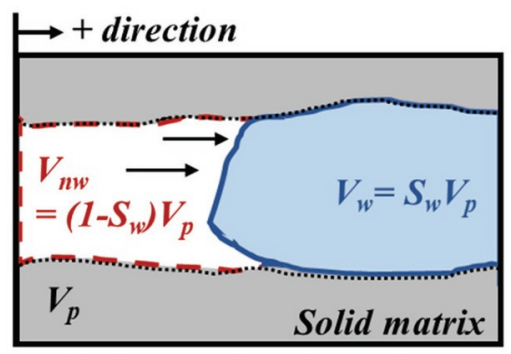

Reference Configuration

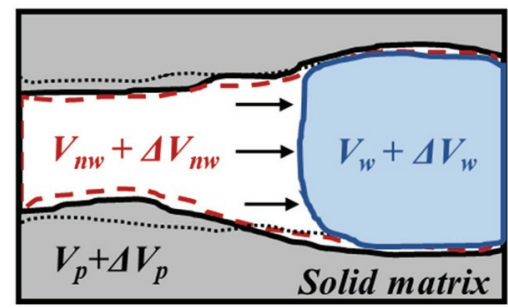

Current Configuration

Fig. 1: Schematic view of injection of nonwetting fluid in the representative element volume with one flow channel. Here, $V_{w}, V_{n w}$, and $V_{p}$ are the volumes of wetting, nonwetting fluids, and pore, respectively. $S_{w}$ is the degree of saturation of wetting fluid.

Partially saturated porous medium experiences a complicated phenomenon that involves three phases and their mechanical, thermal, chemical, and electrochemical interactions. However, the introduced unsaturated poroelastic model only considers the mechanical interaction due to the matric suction effect and limits its validity to the degree of water saturation $\mathrm{Sw}$ from 0.8 to

\footnotetext{
* Corresponding author: hyunbin3@illinois.edu
} 
1.0 to minimize other interactions. In general, the wetting fluid can readily exist as a thin film coating the solid surface by physicochemical nature (van der Waals forces, double-layer repulsion, and chemical cementation) at even lower degrees of saturation.

\section{Results and Discussion}

The water retention curve derived from the mercury intrusion capillary curve is used to cover the wide range of matric suctions (up to $80 \mathrm{MPa}$ ) for the tested remolded and intact specimens of Opalinus Clay. Then, the undrained bulk modulus and Skempton's B value are measured at different degrees of saturation. The unsaturated poroelastic model provides correlation between the degree of saturation $S_{w}$ and $B$ value with other poroelastic constants measured at the fully saturated condition via the knowledge of water retention curve. Figure 2 illustrates the model prediction along the measured B values for intact and remolded shales at degrees of water saturation between 0.8 and 1.0. In general, the model predicts gradual increase in $\mathrm{B}$ value from 0.8 to 1.0 for the soft remolded shale and drastic increase of $\mathrm{B}$ values from 0.2 to 1.0 for the intact shale when the water saturation $S_{w}$ gets above 0.96. These results are not supported by the experimental observations where for both materials, the $\mathrm{B}$ values were monotonically increasing from 0.3 to 1.0 for $S_{w}>0.9$. The model overestimates the effect of desaturation on the unsaturated behavior for the intact material and underestimates it for the remolded one. The discrepancy could be due to neglecting the scale difference between the lab- and microscale properties, but more importantly, the apparent dependency of poroelastic properties from $\mathrm{S}_{\mathrm{w}}$ should be considered. Further studies will concentrate on the modification of unsaturated poroelastic model in terms of the effective stress concept - from Terzaghi's effective stress to suction stress (see, e.g.., Lu and Likos 2006), which might help resolving the overprediction of the impact of nonwetting fluid on shales' response at degrees of saturation above 0.8 .

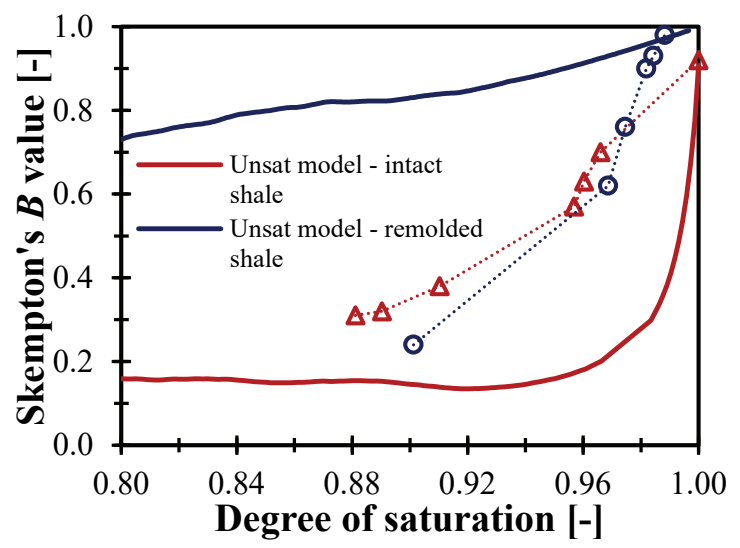

Fig. 2: Variation in Skempton's $B$ value with the degree of water saturation for intact and remolded shale measured in the experiments and predicted from the poroelastic model for unsaturated materials.

\section{References}

1. Cheng, A. (2019). “A linear constitutive model for unsaturated poroelasticity by micromechanical analysis." International Journal of Numerical and Analytical Methods in Geomechanics. 44, 455-483.

2. Lu, N. and Likos, W.J. (2006). "Suction stress characteristic curve for unsaturated soil." Journal of Geotechnical and Geoenvironmental Engineering. 132(2), 131-142. 